\title{
Correction to: An Approximate Augmented Lagrangian Method for Nonnegative Low-Rank Matrix Approximation
}

\section{Hong $\mathrm{Zhu}^{1}$ (D) Michael K. $\mathrm{Ng}^{2} \cdot$ Guang-Jing Song ${ }^{3}$ \\ Received: 5 September 2021 / Accepted: 30 October 2021 / Published online: 7 December 2021 \\ (c) Springer Science+Business Media, LLC, part of Springer Nature 2021 \\ Correction to: Journal of Scientific Computing (2021) 88:45 https://doi.org/10.1007/s10915-021-01556-2}

The original version of this article [4] unfortunately contained an error. The authors would like to correct the error with this corrigendum.

In [4], the optimization formulation is not presented correctly. We should use the following model:

$$
\min _{X} \Phi(X):=\frac{1}{2}\|A-X\|_{F}^{2} \quad \text { subject to } \operatorname{rank}(X) \leq r, X \geq 0 .
$$

instead of (2) stated in [4] to avoid rank-deficient iteration points caused by the fact that the constraint set $\mathcal{M}_{r}=\left\{X \in \mathbb{R}^{m \times n} \mid \operatorname{rank}(X)=r\right\}$ used in [4] is not closed.

In order to derive the Augmented Lagrangian (AL) method for (1), we need to add some geometric properties of $\mathcal{M}_{\leq r}=\left\{X \in \mathbb{R}^{m \times n} \mid \operatorname{rank}(X) \leq r\right\}$ which is a real-algebraic variety [3]. In the rank-deficient point $X \in \mathcal{M}_{\leq r}$ with $\operatorname{rank}(X)=s<r$, the tangent cone $T_{X} \mathcal{M}_{\leq r}$ is given by [2]

$$
T_{X} \mathcal{M}_{\leq r}=T_{X} \mathcal{M}_{s} \oplus\left\{\Xi_{r-s} \in U^{\perp} \otimes \mathcal{V}^{\perp} \mid \operatorname{rank}\left(\Xi_{r-s}\right) \leq r-s\right\},
$$

where $T_{X} \mathcal{M}_{s}$ is the tangent space of $\mathcal{M}_{s}$ at $X, \oplus$ denotes direct sum and $\otimes$ denotes Kronecker product. Then for any $Z \in \mathbb{R}^{m \times n}$, the orthogonal projection of $Z$ onto $T_{X} \mathcal{M}_{\leq r}$ follows

$$
\mathcal{P}_{T_{X} \mathcal{M}_{\leq r}}(Z)=\mathcal{P}_{T_{X} \mathcal{M}_{s}}(Z)+\Xi_{r-s},
$$

The original article can be found online at https://doi.org/10.1007/s10915-021-01556-2.

$凶 \quad$ Michael K. Ng

mng@maths.hku.hk

Hong Zhu

zhuhongmath@126.com

Guang-Jing Song

sgjshu@163.com

1 School of Mathematical Sciences, Jiangsu University, Zhenjiang 212013, Jiangsu, People's

Republic of China

2 Department of Mathematics, The University of Hong Kong, Pokfulam, Hong Kong

3 School of Mathematics and Information Sciences, Weifang University, Weifang 261061, People's Republic of China 

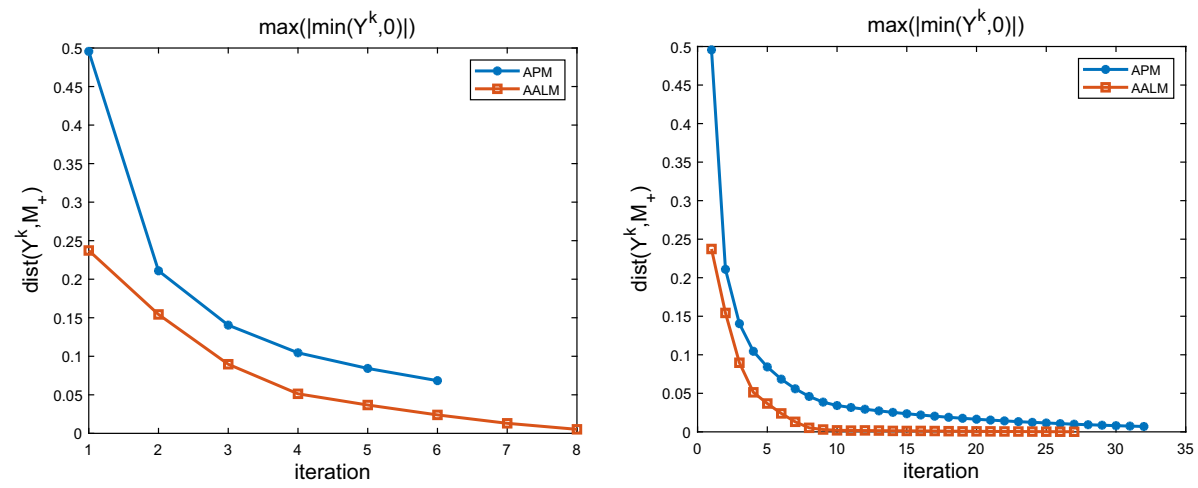

Fig. 1 Correction to Figure 1 in [4]. Iteration convergence on $\operatorname{dist}\left(Y^{k}, \mathcal{M}_{+}\right)$for face dataset UMist with Gaussian noise $\mathcal{N}\left(0,(30 / 255)^{2}\right)$. Left: $\left(\varepsilon_{p}, \varepsilon_{f}\right)=\left(e^{-7}, e^{-9}\right)$; right: $\left(\varepsilon_{p}, \varepsilon_{f}\right)=\left(e^{-10}, e^{-13}\right)$

where $\Xi_{r-s}$ is a best rank- $(r-s)$ approximation of $Z-\mathcal{P}_{T_{X} \mathcal{M}_{r}}(Z)$ in the Frobenius norm. For differentiable function $\Phi$, the critical point $X^{*}$ of $\min _{X \in \mathcal{M}_{\leq r}} \Phi(X)$ satisfies [3]

$$
0=\left\|\nabla \Phi\left(X^{*}\right)\right\|^{2}-\operatorname{dist}\left(-\nabla \Phi\left(X^{*}\right), T_{X^{*}} \mathcal{M}_{\leq r}\right)^{2}
$$

where

$$
\operatorname{dist}\left(Z, T_{X^{*}} \mathcal{M}_{\leq r}\right)=\left\|Z-\mathcal{P}_{T_{X^{*}} \mathcal{M}_{\leq r}}(Z)\right\|_{F} .
$$

Recall that $\mathcal{P}_{T_{X^{*}} \mathcal{M}_{\leq r}}(Z)$ is a orthogonal projection of $Z$ onto $T_{X^{*}} \mathcal{M}_{\leq r}$, it holds that $\left\|\mathcal{P}_{T_{X^{*}} \mathcal{M}_{\leq r}}\left(-\nabla \Phi\left(X^{*}\right)\right)\right\|^{2}=\left\|-\nabla \Phi\left(X^{*}\right)\right\|^{2}-\left\|-\nabla \Phi\left(X^{*}\right)-\mathcal{P}_{T_{X^{*}} \mathcal{M}_{\leq r}}\left(-\nabla \Phi\left(X^{*}\right)\right)\right\|_{F}^{2}$.

Therefore, (2) is equivalent to

$$
\mathcal{P}_{T_{X^{*}} \mathcal{M}_{\leq r}}\left(-\nabla \Phi\left(X^{*}\right)\right)=0 .
$$

If $\operatorname{rank}\left(X^{*}\right)=r$, according to the definition of $\mathcal{P}_{T_{X^{*}} \mathcal{M}_{\leq r}}(\cdot)$, it holds that $\mathcal{P}_{T_{X^{*}} \mathcal{M}_{r}}\left(-\nabla \Phi\left(X^{*}\right)\right)$ $=0$.

The AL subproblem can be rewritten as

$$
\min _{X, Y} \mathcal{L}_{\rho_{k-1}}\left(X, Y, \Lambda^{k-1}\right)+\delta_{\mathcal{M}_{+}}(X)+\delta_{\mathcal{M}_{\leq r}}(Y) .
$$

According to the above discussion of geometric properties of $\mathcal{M}_{+}$and $\mathcal{M}_{\leq r}$, the stationary point $\left(X_{*}^{k}, Y_{*}^{k}\right)$ of (3) satisfies

$$
\left\{\begin{array}{l}
0 \in \nabla_{X} \mathcal{L}_{\rho_{k-1}}\left(X_{*}^{k}, Y_{*}^{k}, \Lambda^{k-1}\right)+\partial \delta_{\mathcal{M}_{+}}\left(X_{*}^{k}\right) ; \\
0=\left\|\nabla_{Y} \mathcal{L}_{\rho_{k-1}}\left(X_{*}^{k}, Y_{*}^{k}, \Lambda^{k-1}\right)\right\|^{2}-\operatorname{dist}\left(-\nabla_{Y} \mathcal{L}_{\rho_{k-1}}\left(X_{*}^{k}, Y_{*}^{k}, \Lambda^{k-1}\right), T_{Y_{*}^{k}} \mathcal{M}_{\leq r}\right)^{2} .
\end{array}\right.
$$

Then Algorithm 1 of [4] can be revised.

The main convergence result of Theorem 1 for Algorithm 1 stated in the original article [4] still holds. By substituting $\mathcal{M}_{\leq r}$ for $\mathcal{M}_{r}$, the proof is the same except formula (17) in [4] should be replaced by

$$
\left|\left\|\frac{1}{2}\left(Y^{k}-A\right)+\Lambda^{k}\right\|^{2}-\operatorname{dist}\left(-\frac{1}{2}\left(Y^{k}-A\right)-\Lambda^{k}, T_{Y^{k}} \mathcal{M}_{\leq r}\right)^{2}\right| \leq \epsilon_{k} / 2, \forall k \in \mathbb{N} .
$$



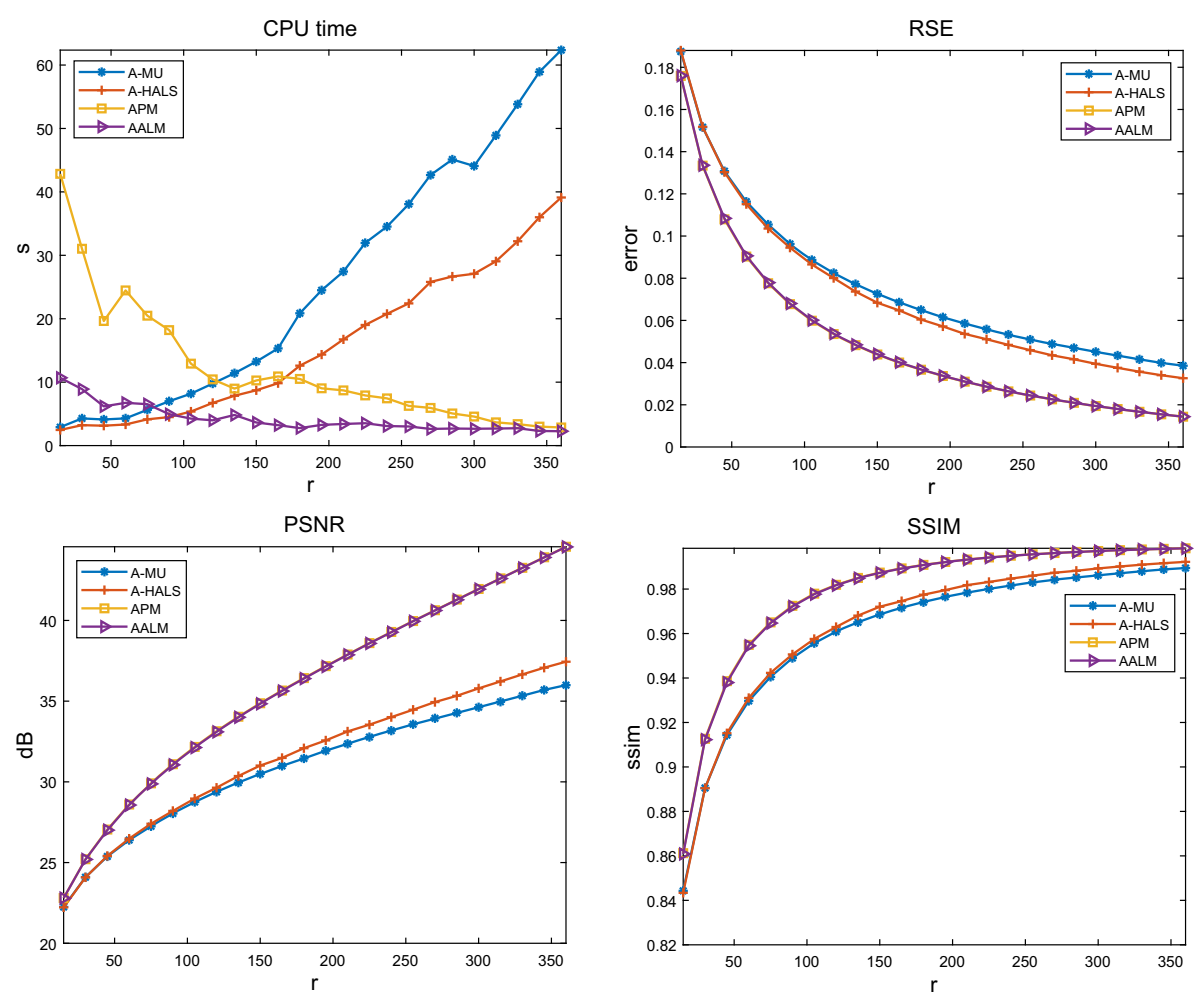

Fig. 2 Correction to Figure 2 in [4]. CPU time, recover qualities RSE, PSNR and SSIM as function of the number of testing rank $r$ for dataset YaleB

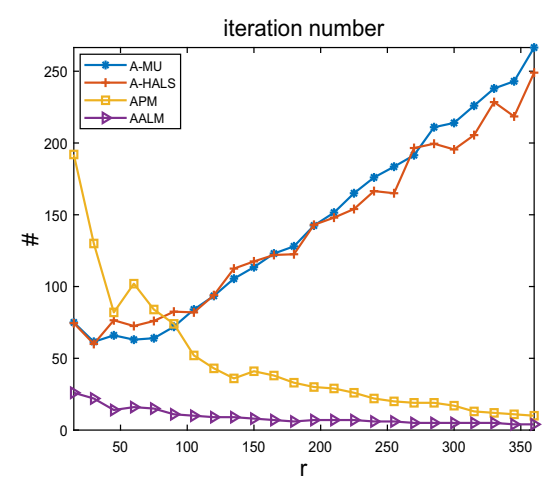

(a) Number of iterations as function of the number of testing rank $r$ for dataset YaleB.

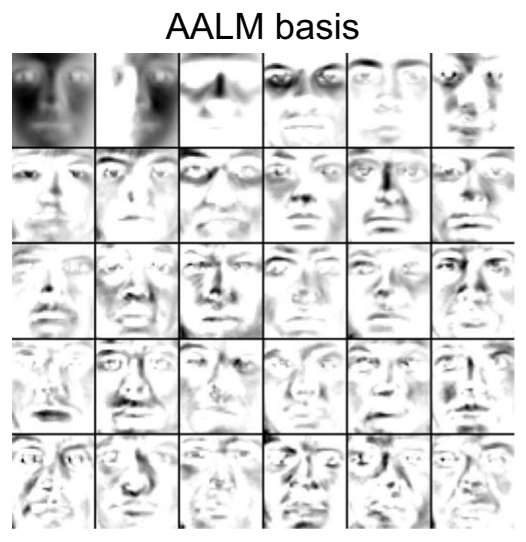

(b) Basis elements obtained by AALM for dataset YaleB.

Fig. 3 Left: correction to Figure 3 in [4]. Right: correction to Figure 5 in [4] 
$\overline{\text { Algorithm } 1 \text { (AALMNLR: Approximate Augmented Lagrangian Method for NLR model. }}$

Require: $\left\{\epsilon_{k}\right\}_{k \in \mathbb{N}} \downarrow 0, \mu>1, k=1, \rho_{0}>0, \theta>0,\left(X^{\text {feasi }}, Y^{\text {feasi }}\right) \in \mathcal{M}_{+} \times \mathcal{M}_{r}, \Lambda^{0} \in \mathbb{R}^{m \times n}$, $\widetilde{\Psi} \geq \max \left\{\Psi\left(X^{\text {feasi }}, Y^{\text {feasi }}\right), \mathcal{L}_{\rho_{0}}\left(X^{\text {feasi }}, Y^{\text {feasi }} ; \Lambda^{0}\right)\right\}$.

Ensure: A sequence $\left\{\left(X^{k}, \Lambda^{k}\right)\right\}_{k \in \mathbb{N}}$.

1: while stopping criterion is not satisfied do

2: $\quad$ Step 1. For given $\rho_{k-1}, \Lambda^{k-1}$, compute $\left(X^{k}, Y^{k}\right)$ for the AL subproblem (3) such that $\left(X^{k}, Y^{k}\right) \in$ $\mathcal{M}_{+} \times \mathcal{M}_{\leq r}$,

$$
\begin{gathered}
\operatorname{dist}\left(-\nabla_{X} \mathcal{L}_{k-1}\left(X^{k}, Y^{k} ; \Lambda^{k-1}\right), \mathcal{N}_{\mathcal{M}_{+}}\left(X^{k}\right)\right) \leq \epsilon_{k} / 2 ; \\
\left|\left\|\nabla_{Y} \mathcal{L}_{k-1}\left(X^{k}, Y^{k} ; \Lambda^{k-1}\right)\right\|_{F}^{2}-\operatorname{dist}\left(-\nabla_{Y} \mathcal{L}_{k-1}\left(X^{k}, Y^{k} ; \Lambda^{k-1}\right), T_{Y^{k}} \mathcal{M}_{\leq r}\right)^{2}\right| \leq \epsilon_{k} / 2 ;
\end{gathered}
$$

and

$$
\mathcal{L}_{\rho_{k-1}}\left(X^{k}, Y^{k}, \Lambda^{k-1}\right) \leq \widetilde{\Psi}
$$

3: $\quad$ Step 2. Update the Lagrangian multipliers

$$
\Lambda^{k}=\Lambda^{k-1}-\rho_{k-1}\left(X^{k}-Y^{k}\right) .
$$

4: Step 3. Update the penalty parameter

$$
\rho_{k}=\max \left\{\mu \rho_{k-1},\left\|\Lambda^{k}\right\|_{\infty}^{1+\theta}\right\}
$$

5: end while

6: return $\left\{\left(X^{k}, \Lambda^{k}\right)\right\}_{k \in \mathbb{N}}$.

Recall (15) and (16) in [4], taking limits as $\mathcal{K} \ni k \rightarrow \infty$ on both sides of (8), there exists $Z^{*} \in \partial \delta_{\mathcal{M}_{+}}\left(X^{*}\right)$, such that

$$
\left\|\nabla \Phi\left(X^{*}\right)+Z^{*}\right\|^{2}-\operatorname{dist}\left(-\nabla \Phi\left(X^{*}\right)-Z^{*}, T_{X^{*}} \mathcal{M}_{\leq r}\right)^{2}=0
$$

which implies that $X^{*}$ is a stationary point of problem (1).

Note that the AL subproblem is a composite optimization problem. It can be checked that (a) $\delta_{\mathcal{M}_{+}}$and $\delta_{\mathcal{M}_{\leq r}}$ are proper lower semicontinuous; (b) $\Theta(X, Y) \triangleq \Psi(X, Y)-\left\langle\Lambda^{k-1}, X-\right.$ $Y\rangle+\frac{\rho_{k-1}}{2}\|X-Y\|_{F}^{2}$ is a $\mathcal{C}^{1}$ function and $\nabla \Theta$ is Lipschitz continuous on bounded subset of $\mathbb{R}^{m \times n} \times \mathbb{R}^{m \times n}$ (for the case that penalty parameter $\rho_{k}$ tends to infinity, the AL subproblem can be scaled by $1 / \rho_{k}$ to ensure that $\nabla_{X} L_{k}(X, Y)$ and $\nabla_{Y} L_{k}(X, Y)$ are Lipschitz continuous.); (c) $\mathcal{M}_{\leq r}$ is a real-algebraic variety [3] and $L_{k}(X, Y)$ is a semi-algebraic function as a finite sum of semi-algebraic functions [1]. Thus the AL subproblem has the KL property. Therefore, the inner-loop solver is valid.

Since the optimality condition of the AL subproblem is changed when substituting $\mathcal{M}_{\leq r}$ for $\mathcal{M}_{r}$, Theorem 2(ii) in [4] should be replaced by (ii) $\left(X^{k, j}, Y^{k, j}\right)$ converges to a critical point of $L_{k}$. Let $\left(X^{k, *}, Y^{k, *}\right)$ be the limit point of $\left\{\left(X^{k, j}, Y^{k, j}\right)\right\}_{j \in \mathbb{N}}$. Then

$$
\begin{aligned}
& 0 \in \nabla_{X} \mathcal{L}_{\rho_{k-1}}\left(X^{k, *}, Y^{k, *}, \Lambda^{k-1}\right)+\partial \delta_{\mathcal{M}_{+}}\left(X^{k, *}\right) ; \\
& 0=\left\|\nabla_{Y} \mathcal{L}_{\rho_{k-1}}\left(X^{k, *}, Y^{k, *}, \Lambda^{k-1}\right)\right\|^{2}-\operatorname{dist}\left(-\nabla_{Y} \mathcal{L}_{\rho_{k-1}}\left(X^{k, *}, Y^{k, *}, \Lambda^{k-1}\right), T_{Y^{k, *}} \mathcal{M}_{\leq r}\right)^{2} .
\end{aligned}
$$


Table 1 Correction to Table 1 in [4]. Average results over 10 tests on synthetic data with fixed $m$

\begin{tabular}{|c|c|c|c|c|c|c|c|}
\hline \multirow[t]{2}{*}{$m$} & \multirow[t]{2}{*}{$n$} & \multirow[t]{2}{*}{$r$} & \multirow[t]{2}{*}{$\sigma^{2}$} & \multicolumn{4}{|c|}{ AALM } \\
\hline & & & & $\mathrm{CPU}$ & RSE & O-ITER & I-ITER \\
\hline \multirow[t]{16}{*}{500} & \multirow[t]{4}{*}{200} & \multirow[t]{4}{*}{10} & 0 & 0.00 & 0.000 & 0 & 0.00 \\
\hline & & & 0.1 & 0.13 & 0.553 & 8 & 1.34 \\
\hline & & & 0.2 & 0.50 & 0.674 & 12 & 2.50 \\
\hline & & & 0.3 & 2.50 & 0.737 & 17 & 10.57 \\
\hline & \multirow[t]{4}{*}{400} & \multirow[t]{4}{*}{20} & 0 & 0.01 & 0.000 & 0 & 0.00 \\
\hline & & & 0.1 & 0.39 & 0.480 & 7 & 1.14 \\
\hline & & & 0.2 & 0.63 & 0.601 & 10 & 1.40 \\
\hline & & & 0.3 & 0.93 & 0.669 & 14 & 1.41 \\
\hline & \multirow[t]{4}{*}{600} & \multirow[t]{4}{*}{30} & 0 & 0.02 & 0.000 & 0 & 0.00 \\
\hline & & & 0.1 & 0.54 & 0.441 & 7 & 1.00 \\
\hline & & & 0.2 & 0.87 & 0.560 & 9 & 1.33 \\
\hline & & & 0.3 & 1.45 & 0.629 & 15 & 1.35 \\
\hline & \multirow[t]{4}{*}{800} & \multirow[t]{4}{*}{40} & 0 & 0.02 & 0.000 & 0 & 0.00 \\
\hline & & & 0.1 & 0.69 & 0.424 & 7 & 1.00 \\
\hline & & & 0.2 & 1.10 & 0.541 & 9 & 1.30 \\
\hline & & & 0.3 & 1.87 & 0.610 & 15 & 1.26 \\
\hline \multirow[t]{16}{*}{1000} & \multirow[t]{4}{*}{200} & \multirow[t]{4}{*}{10} & 0 & 0.01 & 0.000 & 0 & 0.00 \\
\hline & & & 0.1 & 0.35 & 0.557 & 12 & 1.18 \\
\hline & & & 0.2 & 0.41 & 0.679 & 16 & 1.15 \\
\hline & & & 0.3 & 0.74 & 0.743 & 24 & 1.13 \\
\hline & \multirow[t]{4}{*}{400} & \multirow[t]{4}{*}{20} & 0 & 0.02 & 0.000 & 0 & 0.00 \\
\hline & & & 0.1 & 0.76 & 0.498 & 9 & 1.23 \\
\hline & & & 0.2 & 1.17 & 0.621 & 12 & 1.28 \\
\hline & & & 0.3 & 2.10 & 0.689 & 24 & 1.22 \\
\hline & \multirow[t]{4}{*}{600} & \multirow[t]{4}{*}{30} & 0 & 0.04 & 0.000 & 0 & 0.00 \\
\hline & & & 0.1 & 1.42 & 0.459 & 8 & 1.26 \\
\hline & & & 0.2 & 2.28 & 0.581 & 12 & 1.31 \\
\hline & & & 0.3 & 4.29 & 0.651 & 23 & 1.28 \\
\hline & \multirow[t]{4}{*}{800} & \multirow[t]{4}{*}{40} & 0 & 0.08 & 0.000 & 0 & 0.00 \\
\hline & & & 0.1 & 2.20 & 0.434 & 7 & 1.29 \\
\hline & & & 0.2 & 3.61 & 0.555 & 10 & 1.45 \\
\hline & & & 0.3 & 7.09 & 0.625 & 23 & 1.33 \\
\hline
\end{tabular}

By substituting $\mathcal{M}_{\leq r}$ for $\mathcal{M}_{r}$, the stopping criterion for AALM (i.e., formula (19) in the original article [4]) should be replaced by

$$
\left\{\begin{array}{l}
\left\|X^{k}-Y^{k}\right\|<\varepsilon, \\
\left\|\mathcal{P}_{T_{X^{k}} \mathcal{M}_{+}}\left(\frac{1}{2}\left(X^{k}-A\right)-\Lambda^{k}\right)\right\|<\varepsilon, \\
\left|\left\|\frac{1}{2}\left(Y^{k}-A\right)+\Lambda^{k}\right\|^{2}-\operatorname{dist}\left(-\frac{1}{2}\left(Y^{k}-A\right)-\Lambda^{k}, T_{Y^{k}} \mathcal{M}_{\leq r}\right)^{2}\right|<\varepsilon .
\end{array}\right.
$$

Notice that both projections onto $\mathcal{M}_{r}$ and $\mathcal{M}_{\leq r}$ can be obtained by SVD. The only difference between solving the AL subproblem over $\mathcal{M}_{r}$ and $\mathcal{M}_{\leq r}$ occurs when the rank of iteration point is less than $r$. We have retested numerical experiments listed in [4]. Rank- 
Table 2 Correction to Table 2 in [4]. Average results over 10 tests on synthetic data with fixed $n$

\begin{tabular}{|c|c|c|c|c|c|c|c|}
\hline \multirow[t]{2}{*}{$m$} & \multirow[t]{2}{*}{$n$} & \multirow[t]{2}{*}{$r$} & \multirow[t]{2}{*}{$\sigma^{2}$} & \multicolumn{4}{|c|}{ AALM } \\
\hline & & & & $\mathrm{CPU}$ & RSE & O-ITER & I-ITER \\
\hline \multirow[t]{16}{*}{500} & \multirow[t]{4}{*}{200} & \multirow[t]{4}{*}{10} & 0 & 0.00 & 0.000 & 0 & 0.00 \\
\hline & & & 0.1 & 0.13 & 0.553 & 10 & 1.11 \\
\hline & & & 0.2 & 0.18 & 0.674 & 10 & 1.43 \\
\hline & & & 0.3 & 2.29 & 0.737 & 15 & 10.41 \\
\hline & \multirow[t]{4}{*}{400} & \multirow[t]{4}{*}{20} & 0 & 0.01 & 0.000 & 0 & 0.00 \\
\hline & & & 0.1 & 0.41 & 0.480 & 8 & 1.04 \\
\hline & & & 0.2 & 0.63 & 0.601 & 9 & 1.45 \\
\hline & & & 0.3 & 1.97 & 0.669 & 14 & 2.51 \\
\hline & \multirow[t]{4}{*}{600} & \multirow[t]{4}{*}{30} & 0 & 0.02 & 0.000 & 0 & 0.00 \\
\hline & & & 0.1 & 0.56 & 0.438 & 7 & 1.03 \\
\hline & & & 0.2 & 0.91 & 0.556 & 8 & 1.35 \\
\hline & & & 0.3 & 2.90 & 0.625 & 16 & 2.33 \\
\hline & \multirow[t]{4}{*}{800} & \multirow[t]{4}{*}{40} & 0 & 0.02 & 0.000 & 0 & 0.00 \\
\hline & & & 0.1 & 0.62 & 0.410 & 6 & 1.00 \\
\hline & & & 0.2 & 1.05 & 0.526 & 8 & 1.33 \\
\hline & & & 0.3 & 1.88 & 0.595 & 15 & 1.28 \\
\hline \multirow[t]{16}{*}{1000} & \multirow[t]{4}{*}{200} & \multirow[t]{4}{*}{10} & 0 & 0.01 & 0.000 & 0 & 0.00 \\
\hline & & & 0.1 & 0.34 & 0.561 & 8 & 1.38 \\
\hline & & & 0.2 & 0.50 & 0.683 & 11 & 1.43 \\
\hline & & & 0.3 & 11.76 & 0.746 & 18 & 23.89 \\
\hline & \multirow[t]{4}{*}{400} & \multirow[t]{4}{*}{20} & 0 & 0.02 & 0.000 & 0 & 0.00 \\
\hline & & & 0.1 & 0.68 & 0.493 & 8 & 1.08 \\
\hline & & & 0.2 & 1.17 & 0.617 & 10 & 1.47 \\
\hline & & & 0.3 & 7.15 & 0.685 & 14 & 5.56 \\
\hline & \multirow[t]{4}{*}{600} & \multirow[t]{4}{*}{30} & 0 & 0.04 & 0.000 & 0 & 0.00 \\
\hline & & & 0.1 & 1.21 & 0.465 & 8 & 1.03 \\
\hline & & & 0.2 & 2.04 & 0.587 & 9 & 1.47 \\
\hline & & & 0.3 & 3.02 & 0.657 & 13 & 1.53 \\
\hline & \multirow[t]{4}{*}{800} & \multirow[t]{4}{*}{40} & 0 & 0.07 & 0.000 & 0 & 0.00 \\
\hline & & & 0.1 & 1.74 & 0.434 & 7 & 1.06 \\
\hline & & & 0.2 & 3.04 & 0.555 & 9 & 1.39 \\
\hline & & & 0.3 & 9.55 & 0.625 & 13 & 2.54 \\
\hline
\end{tabular}

deficient has not been observed when the rank of the initial point equals $r$. Hence, the performance of the revised algorithm is similar to that in [4]. The revised numerical results are given in Figs. 1, 2, 3, and Tables 1, 2, 3, 4 corresponding to the update in the figures and tables of [4]. 
Table 3 Correction to Table 4 in [4]. Average results for Face Datasets with different $r$

\begin{tabular}{|c|c|c|c|c|c|c|c|c|}
\hline Datasets & YaleB & & & & & & & \\
\hline $\mathrm{r}$ & 75 & & 90 & & 105 & & 120 & \\
\hline alg. & $\mathrm{CPU}$ & RSE & $\mathrm{CPU}$ & RSE & $\mathrm{CPU}$ & RSE & $\mathrm{CPU}$ & RSE \\
\hline AALM & 5.65 & 0.078 & 4.30 & 0.068 & 3.97 & 0.060 & 3.60 & 0.054 \\
\hline Datasets & ORL & & & & & & & \\
\hline $\mathrm{r}$ & 20 & & 30 & & 40 & & 50 & \\
\hline alg. & $\mathrm{CPU}$ & RSE & $\mathrm{CPU}$ & RSE & CPU & RSE & CPU & RSE \\
\hline AALM & 6.55 & 0.176 & 5.61 & 0.159 & 4.40 & 0.147 & 3.82 & 0.138 \\
\hline Datasets & CBCL & & & & & & & \\
\hline $\mathrm{r}$ & 19 & & 29 & & 39 & & 49 & \\
\hline alg. & $\mathrm{CPU}$ & RSE & $\mathrm{CPU}$ & RSE & CPU & RSE & CPU & RSE \\
\hline AALM & 1.48 & 0.120 & 1.34 & 0.099 & 1.11 & 0.085 & 1.24 & 0.074 \\
\hline Datasets & UMsit & & & & & & & \\
\hline $\mathrm{r}$ & 20 & & 40 & & 60 & & 80 & \\
\hline alg. & CPU & RSE & $\mathrm{CPU}$ & RSE & $\mathrm{CPU}$ & RSE & $\mathrm{CPU}$ & RSE \\
\hline AALM & 23.48 & 0.187 & 15.82 & 0.144 & 8.80 & 0.121 & 7.83 & 0.106 \\
\hline Datasets & Olivetti & & & & & & & \\
\hline $\mathrm{r}$ & 10 & & 20 & & 30 & & 40 & \\
\hline alg. & $\mathrm{CPU}$ & RSE & $\mathrm{CPU}$ & RSE & CPU & RSE & CPU & RSE \\
\hline AALM & 2.72 & 0.143 & 2.48 & 0.118 & 2.00 & 0.104 & 1.83 & 0.094 \\
\hline Datasets & Frey & & & & & & & \\
\hline $\mathrm{r}$ & 20 & & 30 & & 40 & & 50 & \\
\hline alg. & $\mathrm{CPU}$ & RSE & $\mathrm{CPU}$ & RSE & CPU & RSE & CPU & RSE \\
\hline AALM & 1.45 & 0.082 & 1.27 & 0.071 & 1.10 & 0.064 & 0.86 & 0.059 \\
\hline
\end{tabular}




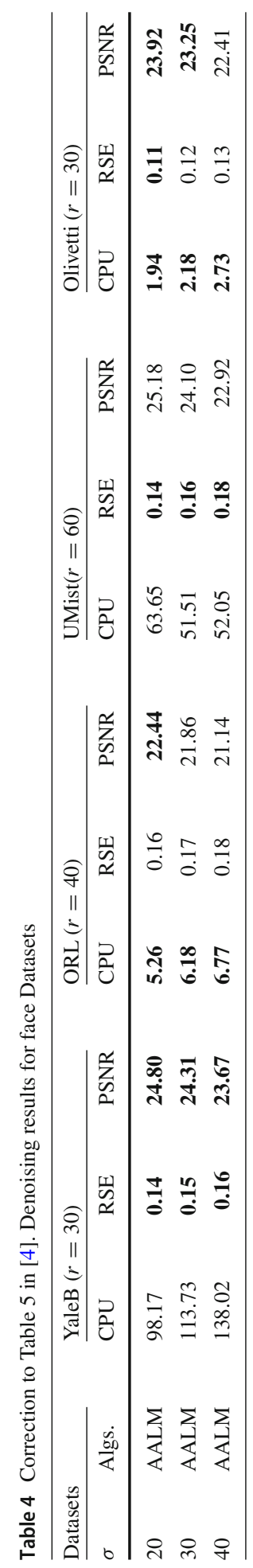




\section{References}

1. Attouch, H., Bolte, J., Redont, P., Soubeyran, A.: Proximal alternating minimization and projection methods for nonconvex problems: an approach based on the Kurdyka-łojasiewicz inequality. Math. Oper. Res. 35(2), 438-457 (2010)

2. Cason, T., Absil, P., Van Dooren, P.: Iterative methods for low rank approximation of graph similarity matrices. Linear Algebra Appl. 438(4), 1863-1882 (2013)

3. Schneider, R., Uschmajew, A.: Convergence results for projected line-search methods on varieties of lowrank matrices via lojasiewicz inequality. SIAM J. Optim. 25(1), 622-646 (2015)

4. Zhu, H., Ng, M., Song, G.: Augmented Lagrangian methods for nonnegative low-rank matrix approximation. J. Sci. Comput. 88 (2021)

Publisher's Note Springer Nature remains neutral with regard to jurisdictional claims in published maps and institutional affiliations. 